\title{
Awareness of Covid-19 and Practice Modification among Dentists during Covid-19 Outbreak
}

\author{
Jeyavel Murugan Jhamunarani ${ }^{1}$, Mohan Narayanan², Ravikumar Thangavel ${ }^{3}$, Karthik Rajaram Mohan ${ }^{4}$ \\ Sabitha Gokul Raj ${ }^{5}$, Saramma Mathew Fenn6, Kumar Appusamy ${ }^{7}$ \\ 1, 2, 3, 4, 5, 6, 7 Department of Oral Medicine and Radiology, Vinayaka Missions Sankarachariar \\ Dental College, Salem, VMRF (DU), TamilNadu, India.
}

\section{ABSTRACT}

\section{BACKGROUND}

Not long after the widespread surge in the corona virus disease 2019 (Covid-19) cases globally, dentistry was identified as one of the riskiest profession during this pandemic. The severe acute respiratory syndrome coronavirus 2 (SARS-CoV-2), similar to many other microorganisms, was found to be prevalent in the mucous and salivary secretions of the infected patients. The proximity of dentists to these bodily fluids demands appropriate enhancement of the safety precautions followed to avoid the spread of infection. During the initial phase of the pandemic lockdowns globally, most dental practices were closed, except for emergency procedures. The fear of the virus was largely prevalent. However, prolonged closures of dental practices with the subsequent halt of all routine and elective dental procedures led to the additional fear of financial losses. This questionnaire study intends to gauge the awareness of Covid19 risks among dentists and evaluate the modifications done in their practices to enhance safety and avoid the spread of infection.

\section{METHODS}

311 dentists participated in an online questionnaire study conducted via google survey. Answers were recorded and statistically analysed using statistical package for social sciences (SPSS).

\section{RESULTS}

97.4 \% of the dentists were aware of Covid-19 while $95.2 \%$ had made at least one or more modifications in their clinical practice to prevent risk of spread of covid-19 viral infection.

\section{CONCLUSIONS}

On the whole, this study implies that majority of the dentists are aware of Covid-19 and have taken some safety precautions to curb the viral spread. In order to ensure that the left out people have sufficient knowledge, help or support, recommendations have been given whenever possible.

\section{KEY WORDS}

Covid-19, Coronavirus, Dentists, Dental Practice Modifications
Corresponding Author: Dr. Jeyavel Murugan Jhamunarani, Department of Oral Medicine and Radiology, Vinayaka Missions Sankarachariar Dental College, Salem, VMRF (DU), TamilNadu, India. E-mail: jeyavelmj@gmail.com

DOI: $10.14260 /$ jemds $/ 2021 / 347$

How to Cite This Article: Jhamunarani JM, Narayanan $M$, Thangavel $R$, et al. Awareness of COVID - 19 and practice modification among dentists during COVID - 19 outbreak. J Evolution Med Dent Sci 2021;10(22):1672-1679, DOI: 10.14260/jemds/2021/347

Submission 01-02-2021, Peer Review 07-04-2021, Acceptance 13-04-2021, Published 31-05-2021.

Copyright (C) 2021 Jeyavel Murugan Jhamunarani et al. This is an open access article distributed under Creative Commons Attribution License [Attribution 4.0 International (CC BY 4.0)] 


\section{BACKGROUND}

The Covid-19 pandemic has completely altered various aspects of our life. In particular, healthcare workers have been identified to have a very high risk of infection due to the proximity to potentially infected patients. Dentists, in particular, have been identified by the world health organization (WHO) as being very prone to these infections due to the presence of the SARS-CoV-2 virus in the saliva and mucosal secretions. ${ }^{1}$

\section{COVID - 19}

The coronavirus disease 2019 was first identified in Wuhan, China, towards the end of 2019. This disease is highly infectious and affects the lungs primarily. The virus SARS$\mathrm{CoV}-2$ ) belonging to the coronavirus family is the aetiologic agent behind this infection. The first symptoms of the Covid19 infection following an incubation period ranging from 2 14 days range from common flu-like symptoms to no symptoms at all. The commonly reported symptoms include fever, cough, breathing difficulty, loss of taste or smell, body pain, malaise, diarrhoea, etc. As the disease advances, the symptoms worsen to pneumonia, sepsis, 'acute respiratory distress syndrome' (ARDS), shock, multi-organ failure, or even death. ${ }^{2}$ To avoid being exposed to this contagion, the use of personal protective equipment such as masks and face shields, frequent hand washing, use of hand sanitizers, and social distancing has been advised by the WHO. An asymptomatic patient exposed to the virus can also be contagious, and hence, these precautions have to be universally implemented to avoid the spread of Covid-19. ${ }^{3}$

\section{Dentists and Covid-19}

During the pandemic, most dentistry related regulatory bodies have advised the suspension of all routine dental procedures. However, all mandatory and emergency dental care visits were allowed, with stringent safety precautions in place.

In a published advisory by the Dental Council Of India (DCI) and the Ministry of Health (Government of India), the two possible modes by which Covid-19 can spread in a dental clinical set up are direct and indirect transmission.

Direct transmission is when someone is infected via direct exposure to infected droplets from the respiratory tract or the oral cavity. The risk from this mode of transmission is high because of the nature of dental procedures, which involves the dental professional's proximity to the oral cavity, exposure to droplets during various dental practices, or when an infected individual coughs, talks, or sneezes. ${ }^{4,5}$

In contrast, indirect transmission or contact transmission is when the person gets infected due to contact with an infected surface or the saliva/mucosal/conjunctival secretions of an infected patient. Additionally, in a dental clinic set up, contact with contaminated surfaces such as the dental chair or instruments can also lead to infection risk. 6

Various studies emphasize the importance of having a well-ventilated setup or space because of the virus' ability to remain suspended in the environment. Millions of droplets released through talking, coughing, sneezing, or routine dental procedures range from $2-3 \mu \mathrm{m}$ in size and can remain on surfaces for over a week. 6.7

Through more effective than mechanical ventilation, natural ventilation can be a critical factor in reducing infection spread if implemented. Protocols by the centre for disease control and prevention (CDC) recommend an average of 12 air exchange cycles to avoid infection, especially if an infected patient is present in the room. Considering that most dental setups are usually closed enclosures, mechanical ventilation should be adopted to ensure health and safety standards are followed.

Spagnuolo et al. suggest that dental professionals should avoid any elective procedure, avoid scheduling of multiple patients in the same time slot or consecutively, reduce waiting time of patients, provide adequate PPE to all staff and patients in the clinic, conduct temperature screening before allowing patients into the clinic and get a thorough medical and travel history from all patients before initiating treatments. ${ }^{8}$

Additionally, dental clinic staff should wear adequate PPE such as the FFP3 masks and maintain physical distancing of 1 m during procedures. Above all, sterilization and disinfection procedures should be carried out religiously. All surfaces should be disinfected before and after every patient or procedure, and mechanical ventilation should follow after every patient visit. ${ }^{9}$

\section{METHODS}

This cross-sectional study was conducted via a google based online survey from October 2020 to December 2020. An Institutional research committee of VMS dental college (from VMRF deemed to be university approved this study. The institutional ethics committee reviewed and approved the study (Approval No. 167 dated - 8/12/2020). This survey was validated by an external team of experienced professors from different universities. The link to this survey was shared on social media to different dentist groups, and the survey data was collected by google forms.

Inclusion criteria comprised all dentists globally who had finished either undergraduate or postgraduate studies in dentistry. The online questionnaire comprised of 22 questions. The first seven questions were general questions that were aimed at gathering necessary information about the respondents. Questions 8 - 12 revolved around the level of awareness related to Covid-19 among the dentists, and questions 12 - 22 were regarding the various modifications done or protocols introduced due to this pandemic. These questions were framed with the current recommendations put forth by multiple organizations such as the World Health Organisation, The Centre for Disease Control and Prevention, The American Dental Association (ADA), The Ministry of Health and Family Welfare MoHFW in India, and the Dental Council of India (DCI).

Key words such as 'Dentists', 'Dental Clinic', 'COVID', 'Coronavirus', 'Practice', 'Modifications', etc. were used in PubMed, Google Scholar and Medscape databases to identify 

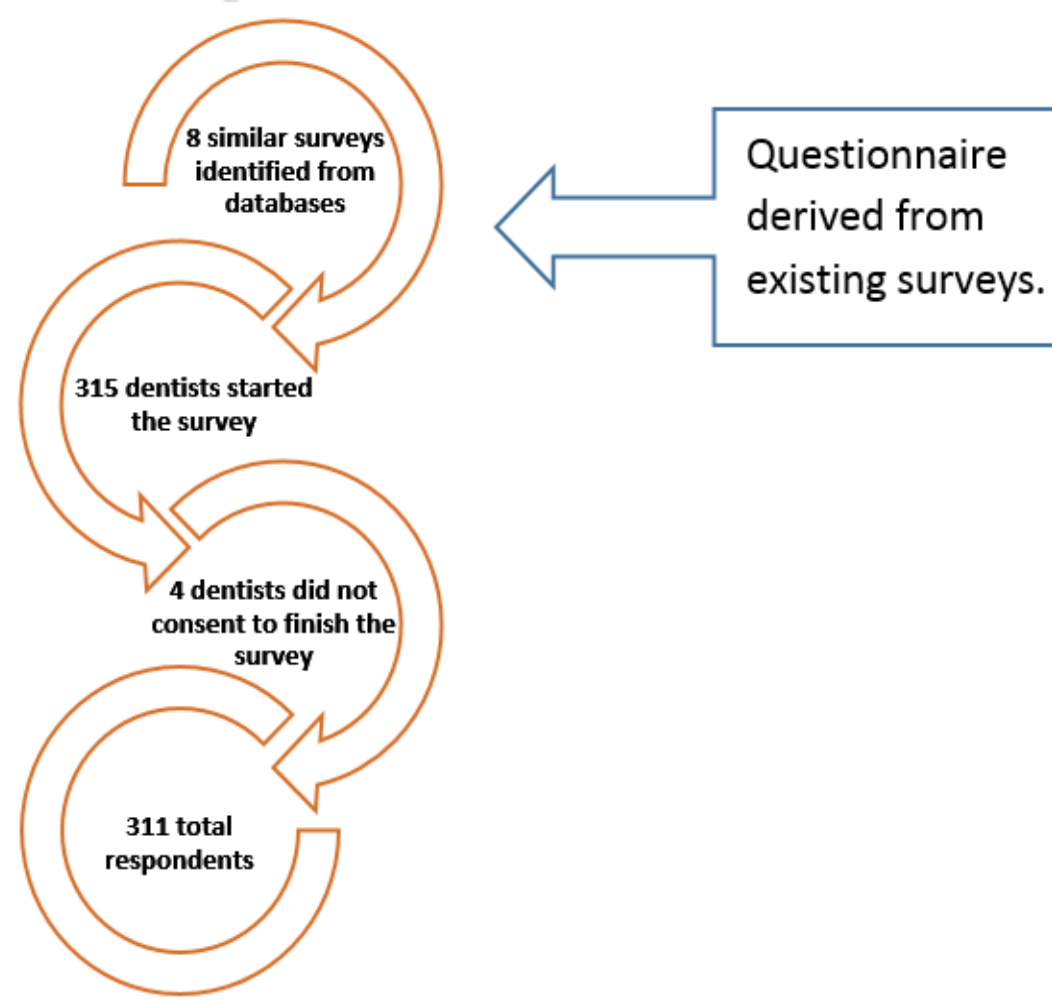

Flowchart 1. Survey Methodology and Inclusion

relevant articles. Flowchart 1 elaborates this further. Strobe checklist was used to report the findings of this survey.

\section{Statistical Analysis}

A total of 311 responses from dentists, mostly located in India, were recorded at the time of drafting this research paper. The data was tabulated in Microsoft excel sheet and the results were statistically analysed using SPSS ver. 23. Percentage analysis was performed to analyse the results descriptively.

\section{RESULTS}

A total of 311 respondents consented to answer the survey, and their answers were thus recorded. Consent was first obtained in the survey form before proceeding further into the survey.

\section{Demographics}

There were a total of 175 females and 136 males in the study. The majority of the respondents were between 26 - 30 years of age (47.3\%), followed by 22 - 25 years (26.7\%), 31 - 40 (18.3\%), 41 - 50 (6.4\%), $51-60(1 \%)$ and above 60 years (0.3\%).

\section{Education Level}

Dentists who held only a bachelor's degree 'BDS' constituted the majority ( $51.4 \%$ ), followed by those with a post graduate 'MDS' (45.7\%), ongoing postgraduate dentists (1.9\%), master's in public health (0.6\%), and finally DDS degree holders $(0.3 \%)$.

\section{Practice Details}

The majority of the respondents were general dentists $(59.5$ $\%$ ), followed by specialty practitioners (20.9\%), consultants (6.4\%), those doing general dentistry and specialty practice (4.8\%), those who were general dentists, specialty practitioners and consultants (3.9\%) and finally those who were general practitioners and consultants (1.9\%). Most of the respondents had less than five years of experience $(63.7$ $\%)$. This was followed by those with 5 - 10 years of experience (21.2\%), 10 - 20 years (11.6\%), and more than 20 years of experience (3.5\%). $84.9 \%$ of the respondents practiced in South India, $11.6 \%$ in North India, and $3.5 \%$ in other countries. Amongst these, $68.2 \%$ of the practice setups were in the urban areas, while $31.8 \%$ were in rural areas.

\section{Awareness of Covid-19}

$97.4 \%$ of the dentists were aware of Covid-19 and its symptoms, while $2.3 \%$ answered that they were not aware of it. $0.3 \%$ responded that they were partly aware of the symptoms. Among the 311 dentists who responded, $2.6 \%$ 


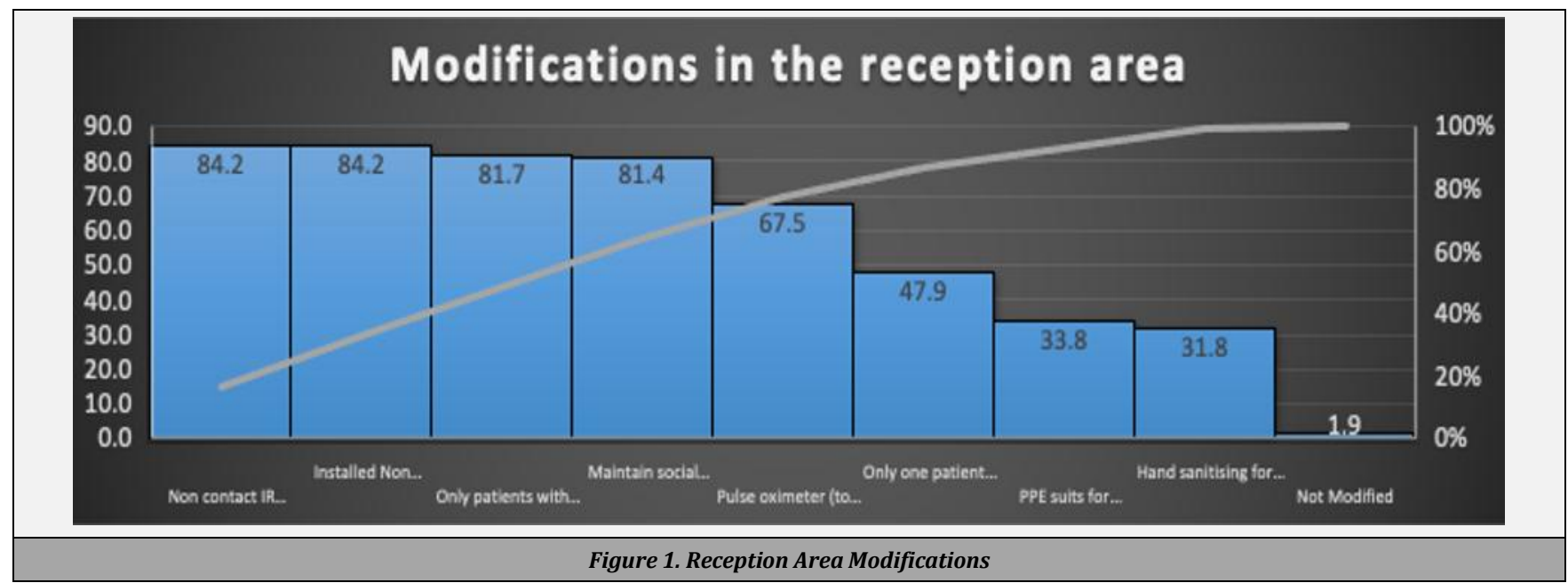

answered that Covid-19 infection spreads through body fluids. In contrast, $5.1 \%$ responded that by close contact with an infected person, $17.7 \%$ responded that droplets or aerosol were responsible. The majority (74.6\%) answered that all of these above could spread infection.

In a dental practice, the source of Covid-19 infection was thought to be due to blood splatter (1.9\%), saliva (2.3\%), aerosol (18.3\%), and all of these (77.5\%). $94.9 \%$ of dentists were well aware that they can get Covid-19 from any patient or attendee, whereas $3.9 \%$ were not sure, and $1.3 \%$ were unaware of this.

\section{Practice Modifications}

Management of $O P$

$35.7 \%$ of the dentists were managing their OPs by taking up patients only by appointments, $31.5 \%$ performed emergency procedures only following an appointment, $19 \%$ provided teleconsultation prior to fixing an appointment, $9 \%$ treated all patients walking in to the clinic, $3.9 \%$ had closed their clinics while $0.9 \%$ were either not practicing dentistry or were not working at any clinic temporarily or triaging patients before initiating treatment.

\section{Reception Area Modifications (Figure 1)}

Almost all dentists had started asking patients to hand sanitize and introduced hand sanitization protocols (84.2\%). Other modifications included using a non-contact IR thermometer $(84.2 \%)$ and pulse oximeter $(67.5 \%)$. Installing a non-contact sanitizer dispenser (84.2\%), maintaining social distancing in waiting areas $(81.4 \%)$, allowing only patients with masks inside the clinic $(81.7 \%)$, allowing only one patient inside reception area (47.9\%), and providing PPE suits for receptionists (33.8\%). $1.9 \%$ of the respondents had not made any modifications at all. This is elaborated in Figure 1

\section{Pre - Procedural Protocols}

$4.8 \%$ of dentists had answered that they had adopted no new protocols before initiating dental procedures. However, a majority (95.2\%) had incorporated either one of the below -
- $\quad$ Asking patients to fill a Covid-19 questionnaire (53.1\%)

- Asking patients to rinse/gargle their mouth with povidone-iodine, $1 \%$ hydrogen peroxide, or a combination of both $(36.3 \%, 20.3 \%$ and $46.3 \%$ respectively). Figure 2 elaborates the findings in detail

\section{Measures Taken in the Operating Area}

Apart from $6.1 \%$ of respondents who had taken no extra measures in their operating area, other dentists had at least taken one of the following measures -

- Installed a new class B or C autoclave (14.8\%),

- Increasing air ventilation (74.9\%),

- Installed a high vacuum suction (18\%),

- $\quad$ Started using negative ion generator (4.5\%),

- Installing a HEPA 13/14 air filter (27.3\%),

- Installing a high flow exhaust fan (27.3\%),

- Installation of UV C light for sterilization of surfaces (25.1 $\%$ ) or

- $\quad$ Stopping air conditioner usage $(71.7 \%)$.

Some dentists were already using some of the above measures prior to the pandemic but their usage has been considerably increased during this pandemic outbreak. And the majority of them have stopped using the air conditioner. Figure 3 elaborates the findings

\section{Treatment Protocols (Figure 4)}

Most respondents (98.7 \%) had taken either one or a combination of the below protocol measure:

- Carrying out only consultations (16.1\%),

- Installing devices for digital impression taking (5.5\%)

- Using PPE such as face shield, masks, and gown for consultation and treatment $(62.7 \%)$

- Using a rubber dam for all procedures (27.7\%) or

- Carrying out only non - aerosol procedures (42.4\%). This is elaborated in Figure 4.

\section{Protective Gear Modifications (Figure 5)}

$2.6 \%$ of the dentists did not modify their existing protective gear usage protocols. However, the majority (97.4\%) carried out one or multiple modifications such as - 
- Using a 3 ply surgical mask for the operator or the assistant or both $(5.1 \%, 7.4 \%$ and $33.8 \%$ respectively). $16.1 \%$ were already using surgical masks for the operator and assistant pre-covid too.

- Using goggles as an eye cover (67.5\%)

- Using a respirator mask with an N95 filter (35\%)

- $\quad$ N95 mask for operator or assistant or both (15.8\%, 9.3 $\%$ and $71.7 \%$ ) Figure 5 elaborates this further.

\section{Post-Treatment Protocols}

$1.9 \%$ respondents did not incorporate any post - treatment protocols. The other $98.1 \%$ incorporated at least one of the following -

- $\quad$ Changing PPE after every patient (29.3\%)

- Cleaning the spittoons after each patient (69.5\%).

- $\quad$ Disinfecting the operating area (82.6\%).

- $\quad$ Fumigating the operating area (34.1\%).

- Mopping the floor with $1 \%$ sodium hypochlorite (49.8 $\%)$
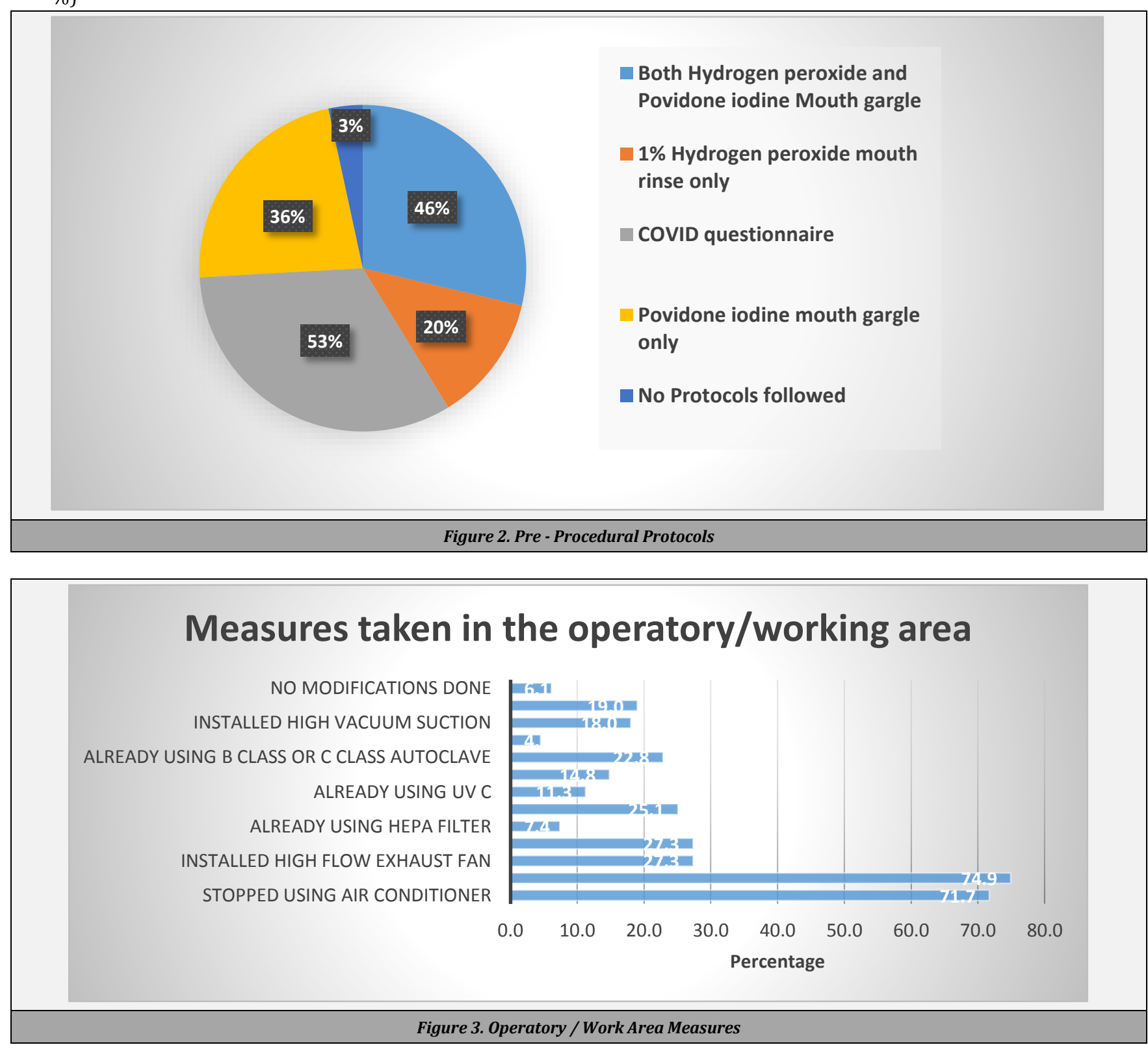

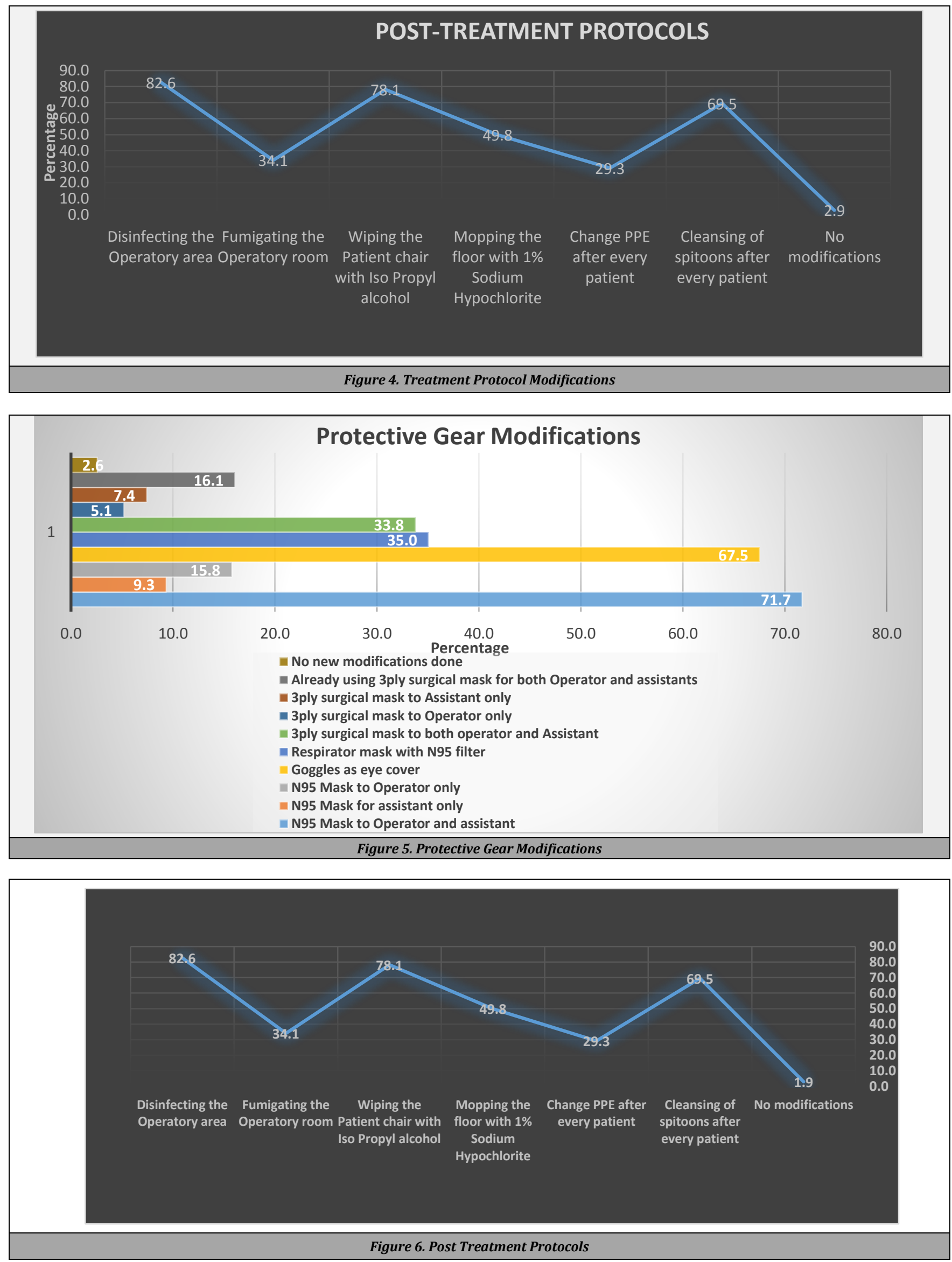


\section{Key Findings / Summary}

1. Majority of the dentists who participated in the survey were aware of COVID-19, its symptoms and precautions needed to prevent infection.

2. Almost all dentists who are currently practising have incorporated at least one or more additional safety measures in their clinics.

3. Commonly adopted changes were pre-procedural changes such as scheduling only emergency appointments, screening of patients before entry into the clinic, providing PPE and hand sanitisers and disinfecting the procedure room periodically.

4. Most common PPE used additionally during the pandemic were eye googles, face shield, gown and respirator with N95 filter.

5. Cleaning spittoons and wiping dental chair with isopropyl alcohol were common practices which were being followed very cautiously and diligently.

6. A significant few had either closed the clinics or were not accepting any appointments indefinitely due to the pandemic.

7. Dentists were already using PPE, autoclaved their instruments regularly and disinfected surfaces in the procedure room. However, all these were being followed more diligently post covid.

8. Allowing air ventilation and avoiding air conditioner usage were commonly practised.

9. Most dentists were using autoclaves but some were not aware of the type of autoclave they have been using.

10. Treatment charges had either been increased or patient was paying separately for the PPE. Overall, most reports point at slightly higher treatment charges for the patient due to this pandemic.

\section{DISCUSSION}

Most dentists were aware of the symptoms of Covid-19. Dentists need to screen patients and look out for symptoms before allowing them to enter the clinic and initiate treatment. This, coupled with knowledge about the multiple ways of viral transmission, is mandated for any practicing dentist. 10 Dentists are expected to follow various protocols put forth by the international or national governing bodies such as the ADA or the DCI to ensure safety of all the staff and patients. ${ }^{11}$ Mouth gargling using antimicrobial solutions such as $20 \mathrm{ml}$ of $1 \%$ hydrogen peroxide is deemed useful in reducing patients' infectivity before initiating the treatment. ${ }^{12}$ Pre and post any procedure. It is recommended that a class - B autoclave and strict sterilization protocols for all hand instruments and rotary devices are followed.13 Using a HEPA 13/14 filter, high volume suction, improving clinic ventilation, using antimicrobials and disinfectants such as $1 \%$ sodium hypochlorite or isopropyl alcohol is mandated by the DCI. ${ }^{14}$ One finding of this study is that most dentists have increased the treatment charges due to this pandemic due to the extra levels of safety measures incorporated and the financial costs of implementing these. Similar to the finding of Ahmed et al. a substantial percentage of dentists had either closed their clinics or did not take up new appointments during the pandemic lockdown periods. ${ }^{15}$
This study also determines that most dentists had a reasonably good knowledge and awareness about Covid-19 and have taken multiple precautionary measures to curb this infection's spread. However, there is still a minority population that is not entirely aware of how Covid-19 can spread and the complete set of precautions needed at the dental clinic to protect themselves, the clinic staff, and the patients from the risk of being infected.

More awareness drives in the form of webinars, pamphlets, emails, etc., should be initiated to ensure dentists in all geographical areas are vigilant and comply with internationally published protocols that can ensure that the maximum safety level and precaution is undertaken in a dental practice.

\section{CONCLUSIONS}

This study revealed that most of the dental practitioners were aware of Covid-19 and their symptoms. This pandemic has brought about many changes in the field of dentistry and paved way for increase in sanitisation protocol which were once only at the level of books in many of the clinics. Considering the above changes, this pandemic could be considered as "the blessing in disguise" which made these good modifications, a mandatory one.

All upcoming dentists should be taught the importance of these protocols right from the undergraduate or postgraduate study levels. Awareness of autoclave types, PPE usage, disinfection procedures etc., should be instilled.

Despite the availability of the vaccine against Covid-19, dentists should continue to follow these protocols and measures to safeguard themselves and their patients from Covid-19 until the pandemic comes under control and also to prevent against any other infection in the future.

This study can be repeated at various stages till the end of the pandemic to better assess the overall situation and infer the take-home points learnt.

\section{Limitations}

This study consists of only a small sample of dentists, and the majority of the dentists who participated were from India. Hence, the results may not be representative of the overall trends. Also, this survey includes dental practitioners who are not currently practicing.

Furthermore, the Covid-19 pandemic is still ongoing and the recommendations by the regulatory bodies around the world have been dynamic with time. Since this survey was conducted during the start of the pandemic, the findings might not be the same now. Therefore, these survey figures might not be completely accurate considering the above mentioned points.

Data sharing statement provided by the authors is available with the full text of this article at jemds.com.

Financial or other competing interests: None.

Disclosure forms provided by the authors are available with the full text of this article at jemds.com. 


\section{REFERENCES}

[1] Fini MB. What dentists need to know about COVID-19. Oral Oncol 2020;105:104741.

[2] Lai CC, Shih TP, Ko WC, et al. Severe acute respiratory syndrome coronavirus 2 (SARS - CoV - 2) and corona virus disease-2019 (COVID-19): the epidemic and the challenges. Int J Antimicrob Agents 2020;55(3):105924.

[3] IDA (2020) https://webcache.googleusercontent.com /search?q=cache:UmFD1i7HFZgJ:https://www.ida.org.i n/pdf/IDA_Recommendations_for_Dental_Professionals_ on_the_Coronavirus_Threat.pdf $+\& \mathrm{~cd}=8 \& \mathrm{hl}=\mathrm{en} \& \mathrm{ct}=\mathrm{clnk} \&$ $\mathrm{gl}=\mathrm{in}$

[4] Cumbo E, Gallina G, Messina P, et al. Alternative methods of sterilization in dental practices against COVID-19. Int J Environ Res Public Health 2020;17(16):5736.

[5] World Health Organization. Coronavirus disease (COVID19): situation report 2020: p. 171.

[6] Fiorillo L, Cervino G, Matarese M, et al. COVID-19 surface persistence: a recent data summary and its importance for medical and dental settings. Int J Environ Res Public Health 2020;17(9):3132.

[7] Coulthard P. Dentistry and coronavirus (COVID-19)moral decision-making. Br Dent J 2020;228(7):503-5.

[8] Spagnuolo G, De Vito D, Rengo S, etal. COVID-19 outbreak: an overview on dentistry. Int J Environ Res Public Health 2020;17(6):2094.
[9] Rahman HS, Aziz MS, Hussein RH, et al. The transmission modes and sources of COVID-19: a systematic review. International Journal of Surgery Open 2020;26:125-36.

[10] Diegritz C, Manhart J, Bücher K, et al. A detailed report on the measures taken in the department of conservative dentistry and periodontology in munich at the beginning of the COVID-19 outbreak. Clin Oral Investig 2020;24(8):2931-41.

[11] Raman P. Holistic approach on dentistry post COVID-19critical assessment of literature. International Journal of Recent Advances in Multidisciplinary Research 2020;07(07):6049-56.

[12] Burton MJ, Clarkson JE, Goulao B, et al. Use of antimicrobial mouthwashes (gargling) and nasal sprays by healthcare workers to protect them when treating patients with suspected or confirmed COVID-19 infection. Cochrane Database of Syst Rev 2020;9:CD013626.

[13] Hegde MN, Qaiser S, Hegde ND. Clinical protocols in dental practice: post-COVID-19. J Conserv Dent 2019;22(5):40810.

[14] Kumar GA, Mohan R, Hiremutt DRP, et al. COVID-19 pandemic and safe dental practice: need of the hour. Journal of Indian Academy of Oral Medicine and Radiology 2020;32(2):164-71.

[15] Ahmed MA, Jouhar R, Ahmed N, et al. Fear and practice modifications among dentists to combat Novel Coronavirus Disease (COVID-19) outbreak. Int J Environ Res Public Health 2020;17(8):2821. 